\title{
Drug Induced Immune-Thrombocytopenia
}

Immune-thrombocytopenia caused by drugs has fascinated hematologists, internists and other investigators for more than 30 years. A number of elaborate in vitro tests based on different principles have been developed. These methods have been derived partly from experience in bacteriology, such as platelet agglutination, lysis and complement fixation, or from the blood coagulation process and immunology such as clot retraction inhibition, platelet factor 3 immuno-injury test, 51Cr platelet lysis test, release (or inhibition of uptake) of serotonin, platelet aggregometry, serologic-nephelo-metric tests, lymphocyte transformation, etc. (The last two tests are not specifically related to the thrombocyte as the mainly involved cell of the allergic drug reaction.)

Three papers in this journal (vol. 68, pp. 68, 74 and 75) deal with drug-induced thrombocytopenic purpura and add two more causative drugs, namely carbama-zepine (Tegretol ${ }^{\circledR}$ ) and the beta blocking agent oxprenolol (Trasitensin ${ }^{\circledR}$ ). These two agents have hardly been known to provoke this syndrome by an allergic or immunolog-ic mechanism before. One of the most important and practical problems in drug-induced thrombocytopen-ias is the identification of the responsible drug, as follows.

The Possibility to Identify the Causative Drug by in vitro Tests

Most of the in vitro tests are either not very sensitive or difficult to perform and therefore not yet a part of routine laboratory studies. They seem to correlate well with the clinical estimation of causality of a given drug, provided they are precisely performed, critically interpreted, and show a positive result.

A negative result, however, does not exclude a suspected drug. Thus, the clinician responsible for the patient is always confronted with the question if it is not best to discontinue all the drugs taken by the patient before the appearance of acute throm-bocytopenia and to substitute chemically non-related preparations. This is especially true for drugs known to occasionally induce thrombocytopenia.

2

Hoigné

The Risk of Drug Reexposure

Reexposure to the suspected drug for verification should be advised only after careful consideration of the risk involved in relation to the importance of the particular drug for the individual patient. In my personal opinion, reexposure of patients with acute thrombocytopenia or agranulocytosis to a drug known to produce this kind of blood dyscrasia is generally a nonacceptable hazard. Experts in the fields of hematol-ogy and drug safety principally have avoided reexposing patients in this situation since the mid-sixties. There is good reason to maintain this principle even in a time of possible thrombocyte and leukocyte replacement! This holds true despite the fact that drug challenge with minute doses has been safely accomplished by some very experienced and critical investigators on a small number of patients. If a drug is suspected for which no convincing evidence of a relationship to thrombocytopenia or agranulocytosis is yet 
available, the problem of exposure to minute doses, possibly even with subsequent dose increase, is open to discussion. In such instances the patient certainly must be informed of the considerable potential risk, and platelet counts should be performed at small time intervals. The results should be published or at least present-

ed to a drug monitoring center collecting adverse drug reactions, which in turn will publish such information. A study of this kind will probably be of greater benefit to expanding medical knowledge than to the individual patient, who has to accept the risk.

Repeated reexposures to the same drug, involving a risk which cannot be reasonably predetermined, is generally not acceptable for any physician or investigator with a sound sense for drug-safety.

The most dangerous adverse drug reactions found in the Comprehensive Hospital Drug Monitoring Program Berne, which controlled 17,285 inpatients of the divisions of internal medicine at two teaching hospitals (by electronic data processing since 1974), are thrombocytopenia, agranulocytosis and gastrointestinal hemorrhage in relation to analgesic/antirheumatic drugs as well as complications of anticoagulants and cardiac glycosides. The cytostatic and immunosuppressive drugs should be considered separately because of the severity of the basic diseases for which they are given.

Prof. Dr. med. R. Hoigné,

Head, Division of Internal Medicine,

Zieglerspital,

CH-3001 Bern (Switzerland) 